\title{
Normal physiology and HIV pathophysiology of human T-cell dynamics
}

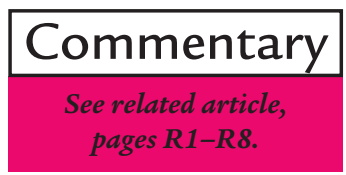

\author{
Douglas D. Richman
}

Departments of Pathology and Medicine - 0679, San Diego Veterans Affairs Medical Center and University of California-San Diego, 9500 Gilman Drive, La Jolla, California 92037-0679, USA.

Phone: (858) 552-7439; Fax: (858) 552-7445; E-mail: drichman@ucsd.edu.

The central feature of HIV disease is opportunistic infection and malignancy resulting from $\mathrm{CD} 4$ cell depletion. The remarkable reversal of this morbidity and mortality among the economically privileged results from the advent of potent antiretroviral therapy that allows the restoration of CD4 cell numbers and function. Insights into HIV dynamics and the relationships between of HIV replication and CD4 cell depletion have revolutionized our understanding of HIV pathogenesis and the clinical management of HIV infection (1-5). This revolution has also unveiled the magnitude of our ignorance about the dynamics of $\mathrm{T}$ lymphocytes in both HIV disease and normal physiology (6).

The introduction of potent antiretroviral therapy and the use of mathematical modeling enabled calculations for HIV clearance rates, HIV production rates, durations of components of the virus replication cycle, viral and infected lymphocyte survival times, and HIV generation time (1-4). Studies of lymphoid tissue permitted measurements of viral dynamics in solid tissue, characterized infected target cells in solid tissue, and confirmed the correlation of virus replication in lymphoid tissue and blood (7-12). Viral dynamics have been investigated not only in the quasi-steady state of established infection, but during primary infection (13).

Why has the dynamics of $\mathrm{T}$ lymphocytes been so difficult to address, relative to HIV viral dynamics, or even to that of erythrocytes and platelets, other blood cells whose turnover and life spans were well defined over a generation ago? The first problem is to measure proliferation rates. Measuring telomerase length is indirect and subject to complicated assumptions $(14,15)$. The measurement of cell-cycle antigens like Ki67 cannot account for variations in phases in the cell cycle. External labels incorporated into replicating T-cell DNA, such as BrdU and deuterated glucose, are not infused instantaneously, require large numbers of cells for detection, and, in the case of BrdU, are subject to recycling of label via salvage pathways $(16,17)$. These methods do not account for cell death during labeling or before measurement. A recent review has lucidly summarized these various methods and their limitations (18).

Another problem, not encountered when working with cells such as erythrocytes or platelets, is that CD4 and CD8 lymphocytes are a remarkably complex and heterogeneous mixture of subpopulations, each with the prospect of different turnover rates and tissue distributions, not to mention differential susceptibility to HIV infection. Furthermore, only $2 \%$ of $\mathrm{T}$ lymphocytes are normally found in the blood, and the distribution of these cells shifts significantly both with HIV infection and its treatment $(10,19,20)$. The distribution of these heterogeneous subpopulations in the lymphoid tissue may not be perfectly reflected in the blood. Worse yet, not all lymphoid tissues are the same. For example, the susceptibility of lymphocytes to lentivirus infection may differ remarkably in the gut and lymph nodes $(21,22)$.

Finally, the modeling and calculations based on these various measurements must make certain assumptions. For example, is cell survival unchanged under different conditions that affect rates of cell proliferation? Is cell survival the same in HIV-infected and uninfected patients even with similar proliferation rates? When examining subpopulations of CD4 and CD8 lymphocytes, what proportion of the changes observed reflect production, death, or shift from one phenotype to another?

Despite these imposing challenges, progress is being made in characterizing T-cell dynamics in HIV infection. Studies to date indicate that CD4 cell depletion is associated with increased T-cell turnover in HIV infection (16, 17, 23); however, the dynamics are not strictly parallel to hemolytic anemia or idiopathic thrombocytopenic purpura, conditions that induce dramatic rises in both cell production and elimination. After HIV infection, the half-life of CD4 T lymphocytes drops, but the production rate does not respond with a compensatory increase $(16,24)$. With the suppression of virus replication by antiretroviral therapy, production rates of both CD4 and CD8 cells increase significantly $(16,21)$. Adding to these changes, HIV infection redistributes lymphocytes from circulation to the solid tissues, and antiretroviral therapy partially reverses this process $(10,20)$.

The article by McCune et al. in this issue of the JCI (25) confirms many of these observations and significantly extends them. The clinically critical decay of CD4 cell numbers in untreated HIV-infected patients is explained by a shortened cellular half-life without

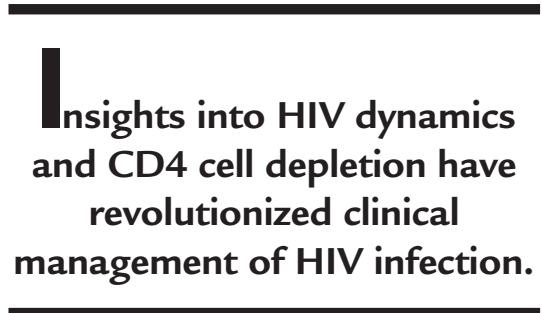

a compensatory increased rate of production. Antiretroviral treatment corrects the CD4 cell count by relieving the inhibition of production but has little effect on the turnover of these cells. After the first year of therapy, however, production rates settle to normal levels and the cellular half-life lengthens. These 2 phases, with a more rapid turnover followed by a slower one, may reflect the different half-lives of lymphocyte subpopulations. Memory/effector cells $\left(\mathrm{CD} 45 \mathrm{RO}^{+}\right)$appear to have a half-life of 1-2 months, whereas naive cells $\left(\mathrm{CD}^{2} 5 \mathrm{RA}^{+}, \mathrm{CD}^{2} \mathrm{~L}^{+}\right)$have half-lives of 4-12 months, as suggested by previous human and murine stud- 
ies. These populations are each yet more complicated with the memory/effector subpopulation consisting of further fractions of memory and effector components with each of these containing specialized members. Naive cells, as well, may be generated by peripheral proliferation or by emigration from the thymus; recent thymic emigrants can be identified because they retain DNA rearrangement circles derived from the T-cell receptor locus (26). McCune et al. show that some of the interpatient variability in CD4 cell production represents differential production of longer half-life, naive T cells, a property that correlates with agerelated restoration of thymic mass. The generation, or lack of it, of each of the many components of the T-cell population undoubtedly has profound effects on the repertoire and function of the immune system.

New insights and information beget yet more unanswered questions. Are the assumptions underlying the mathematical models that generated these determinants all valid? For example, cells eliminated between the times of labeling and assay cannot be accounted for in these models. What are the dynamics, the trafficking, and the distribution of the various subpopulations in different tissue compartments? What mechanisms underlie the modulation of production, survival, phenotypic shifting, trafficking, and redistribution in HIV disease. Finally, what regulates these processes in normal physiology? The study of HIV pathophysiology prodded inves- tigators to address the normal physiology of cell dynamics, but this work has only begun.

\section{Acknowledgments}

This work was supported by grants from the Center for AIDS Research (AI 27670, AI 38858, and AI 36214) and from the National Institutes of Health (AI 29164) and by the Research Center for AIDS and HIV Infection of the San Diego Veterans Affairs Medical Center.

1. Ho, D.D., et al. 1995. Rapid turnover of plasma virions and CD4 lymphocytes in HIV-1 infection. Nature. 373:123-126.

2. Wei, X., et al. 1995. Viral dynamics in human immunodeficiency virus type 1 infection. Nature. 373:117-122.

3. Perelson, A.S., Neumann, A.U., Markowitz, M., Leonard, J.M., and Ho, D.D. 1996. HIV-1 dynamics in vivo: virion clearance rate, infected cell lifetime, and viral generation time. Science. 271:1582-1586.

4. Perelson, A.S., et al. 1997. Decay characteristics of HIV-1-infected compartments during combination therapy. Nature. 387:188-191.

5. Mellors, J.W., et al. 1997. Plasma viral load and CD4 ${ }^{+}$lymphocytes as prognostic markers of HIV1 infection. Ann. Intern. Med. 126:946-954.

6. Hellerstein, M.K., and McCune, J.M. 1997. T cell turnover in HIV-1 disease. Immunity. 7:583-589.

7. Haase, A.T., et al. 1996. Quantitative image analysis of HIV-1 infection in lymphoid tissue. Science. 274:985-989.

8. Cavert, W., et al. 1997. Kinetics of response in lymphoid tissues to antiretroviral therapy of HIV 1 infection. Science. 276:960-964.

9. Wong, J.K., et al. 1997. Reduction of HIV-1 in blood and lymph nodes following potent anti retroviral therapy and the virologic correlates of treatment failure. Proc. Natl. Acad. Sci. USA. 94:12574-12579.

10. Zhang, Z.-Q., et al. 1998. Kinetics of CD4+ T cell repopulation of lymphoid tissues after treatment of HIV-1 infection. Proc. Natl. Acad. Sci. USA 95:1154-1159.

11. Günthard, H.F., et al. 1998. Human immunodeficiency virus replication and genotypic resistance in blood and lymph nodes after a year of potent antiretroviral therapy. J. Virol. 72:2422-2428.

12. Zhang, Z.-Q., et al. 1999. Sexual transmission and propagation of SIV and HIV in resting and activated $\mathrm{CD}^{+} \mathrm{T}$ cells. Science. 286:1353-1357.

13. Little, S.J., McLean, A.R., Spina, C.A., Richman, D.D., and Havlir, D.V. 1999. Viral dynamics of acute HIV-1 infection. J. Exp. Med. 190:841-850.

14. Wolthers, K.C., et al. 1996. T cell telomere length in HIV-1 infection: no evidence for increased CD4+ T cell turnover. Science. 274:1543-1547.

15. Palmer, L.D., et al. 1997. Telomere length, telomerase activity, and replicative potential in HIV infection: analysis of CD4+ and CD8+ T cells from HIV-discordant monozygotic twins. J. Exp. Med. 185:1381-1386

16. Hellerstein, M., et al. 1999. Directly measured kinetics of circulating $\mathrm{T}$ lymphocytes in normal and HIV-1-infected humans. Nat. Med. 5:83-89.

17. Mohri, H., Bonhoeffer, S., Monard, S., Perelson, A.S., and Ho, D.D. 1998. Rapid turnover of T lymphocytes in SIV-infected rhesus macaques. Science. 279:1223-1226.

18. Hellerstein, M.K. 1999. Measurement of T-cell kinetics: recent methodologic advances. Immunol. Today. 20:438-441.

19. Pakker, N.G., et al. 1998. Biphasic kinetics of peripheral blood $\mathrm{T}$ cells after triple combination therapy in HIV-1 infection: a composite of redistribution and proliferation. Nat. Med. 4:208-214.

20. Bucy, R.P., et al. 1999. Initial increase in blood $\mathrm{CD}^{+}$lymphocytes after HIV antiretroviral therapy reflects redistribution from lymphoid tissues. J. Clin. Invest. 103:1391-1398.

21. Veazey, R.S., et al. 1998. Gastrointestinal tract as a major site of $\mathrm{CD}^{+} \mathrm{T}$ cell depletion and viral replication in SIV infection. Science. 280:427-431.

22. Harouse, J.M., Gettie, A., Tan, R.C.H., Blanchard, J., and Cheng-Mayer, C. 1999. Distinct pathogenic sequela in rhesus macaques infected with CCR5 or CXCR4 utilizing SHIVs. Science. 284:816-819.

23. Sachsenberg, N., et al. 1998. Turnover of CD4+ and CD8+ T lymphocytes in HIV-1 infection as measured by Ki-67 antigen. J. Exp. Med. 187:1295-1303.

24. Fleury, S., et al. 1998. Limited CD4+ T-cell renewal in early HIV-1 infection: effect of highly active antiretroviral therapy. Nat. Med. 4:794-801.

25. McCune, J.M., et al. 2000. Factors influencing Tcell turnover in HIV-1-seropositive patients. $J$. Clin. Invest. 105: R1-R8 (2000).

26. Douek, D.C., et al. 1999. Changes in thymic function with age and during the treatment of HIV infection. Nature. 396:690-695. 